\title{
Qualidade da informação nas publicações científicas electrônicas na Internet: desafios e propostas
}

\author{
Marcelo Sabbatini \\ Universidade Metodista de Sâo Paulo \\ sabbatini@webpraxis.com
}

\section{Introdução}

As publicações científicas eletrônicas na Internet [1] constituem atualmente um dos temas de maior repercussão dentro da comunicação científica, e por conseguinte, da própria ciência moderna, ao atuarem dentro de um campo chave para o funcionamento do atual modelo de produção do conhecimento científico, o sistema de publicações científicas. Tais publicações permitem uma maior flexibilidade e variedade de aplicações, em comparação com o sistema tradicional de publicações baseado no papel, com a possibilidade de apresentar uma informação mais rica nos mais diversos sentidos, além de freqüentemente serem anunciadas como uma forma mais barata de publicação, que solucionaria a crise econômica que afeta este campo na atualidade. No entanto, na mesma medida em que as publicações eletrônicas acenam com algumas possíveis melhorias para a comunidade científica, colocam uma série de dificuldades, que poderiam ser resumidas, basicamente, na mudança de práticas e culturas associadas com a publicação científica. Dentre estas questões, que analisaremos a seguir, podemos destacar o problema da certificação de qualidade, da manutenção da integridade e segurança das publicações e mesmo da própria percepção que a comunidade científica teria desta nova forma de comunicação e suas implicações em processos de promocão e avaliação acadêmica.

\section{A revisão por pares}

As questões em torno da natureza, estrutura e valor da avaliação pelos pares pares, enquanto forma de certificação da\&n qualidade acadêmica, tornam-se um ponto crucial na aceitação do modelo eletrônico de publicação, (Langston, 1996). O sistema de análise por pares nas revistas impressas tradicionais funciona de forma simples, porém passível de falhas. O editor, juntamente com o conselho editorial, escolhe árbitros para cada manuscrito submetido, que por sua vez emitem pareceres (algumas vezes anônimos, outras vezes não) recomendando/rejeitando o artigo em questão e sugerindo revisões.Um terceiro árbitro pode ser consultado no caso de situações de impasse. Os relatórios emitidos pelos árbitros possuem caráter de aconselhamento, sendo a decisão final de publicação realizada pelo editor e enviada ao autor, juntamente com os informes dos árbitros. Dentro das respostas possíveis estão a aceitação imediata, acompanhada ou não de comentário elogioso; a devolução dos originais com sugestões de modificações, como por exemplo estilo de redação, adição ou eliminação de dados, modificações das conclusões ou conceitos manipulados e que constitui a situação mais comum e finalmente, recusa do trabalho. No caso da devolução acompanhada de sugestões, o autor pode argumentar razões contrárias às opiniões dos árbitros, iniciando um período de réplicas e contra-réplicas que pode durar vários meses, prejudicando a rapidez de difusão da informação aos grupos interessados. No caso de recusa, o autor podem reenviar o trabalho a outra revista menos solicitada, com menor taxa de rejeição, menos rigorosa, menos prestigiada ou de menor impacto, assim como tentar a sorte em outra revista de maior categoria (Primo-Yúfera, 1994). 
A idéia por trás da análise dos pares é liberar o processo de publicação das preferências de indivíduo particular, tornando-o questionável à comunidade de pares como um todo (Harnad, 1996). Além disso, o sistema de revisão por pares atua como uma ferramenta de convalidação, necessária para o estabelecimento do reconhecimento e dos próprios resultados, dentro da comunidade científica. Esta característica é explicada pelo fato de que a revisão supõe a superação de uma crítica severa, perpetrada por especialistas. Ainda mais, a aceitação das próprias limitações, na forma de erros e desvios, forma parte do processo através do qual os conhecimentos são postos a prova. Também cabe destacar que este sistema de "filtração" funciona como uma resistência à pressão por publicar produzida pelo sistema científico-acadêmico-administrativo. Outra característica positiva deste sistema é o reconhecimento favorável por um grande número de cientistas, que consideram que quase todos os artigos publicados melhoram durante o processo de aceitação, favorecendo a qualidade da pesquisa (Primo-Yúfera, 1994).

Nas ciências sociais e humanidades, as revistas possuem uma tendência de se prestigiarem através da magnitude das taxas de rejeição, não sendo incomum taxas de 80 a 90 por cento de rejeição nas revistas mais prestigiadas. Nas ciências físicas e matemáticas, o prestígio não é associado com taxas de rejeição, e nas revistas das áreas biológica, médica e de engenharia, as taxas de rejeição se situam em uma faixa intermediária (Hargens, 1990). Independentemente do campo, o rigor na rejeição é maior nas revistas de maior prestígio, que têm maior impacto sobre a comunidade científica e que recebem mais artigos para publicação. Este fato promove uma espiral de seleção, com a consequiência de que estas revistas também são as mais citadas.

O ponto falível do sistema de revisão por pares seria não o árbitro e seu julgamento humano, porém a seleção deste árbitro, uma função realizada pelo editor. Os editores geralmente possuem uma reserva de árbitros para cada especialidade. Em áreas ativas, esta população de árbitros pode se mostrar saturada. Um dos resultados do sobreuso dos mesmos árbitros seria a lentidão do processo de revisão, com longos intervalos de tempo sendo requeridos para a leitura, e a emissão dos relatórios pode ser realizada de última hora, não refletindo a avaliação cuidadosa e consciente que o sistema exigiria (Harnad, 1996). Esta situação é agravada pelo fato de que os árbitros geralmente são cientistas importantes, com diversas ocupações de caráter acadêmico, docente e administrativo, fator pelo qual se questiona a dedicação dos árbitros ao processo de revisão. Também se assinalam, como defeitos deste sistema, a disparidade de critérios e a falta de coerência nos informes emitidos pelos árbitros. A maior crítica que se faz atualmente é de que os juízos demonstram vieses em favor de cientistas, universidades e centros de pesquisa de prestígio, e que estaria compreendido de certa forma, dentro do efeito Mateus, uma teoria da sociologia clássica da ciência que postula que aqueles que possuem um certo nível de prestígio acadêmico tem maior facilidade de serem escutados e valorizados. Recebem mais meios, mais atenção e mais facilidade de aumentar o prestígio, resultando em uma retroalimentação do êxito social (Merton, 1968).

Assim, o processo tradicional parece passar por uma crise metodológica, com a observação da existência de falhas, como a não detecção de erros em artigos científicos e a inutilidade do ocultamento da identidade dos revisores e autores, e vieses, como a seleção de trabalhos conforme a avaliação de parâmetros como a posição acadêmica, currículo científico, e sexo do pesquisador, além da a predominância geográfica e lingüística da ciência anglo-saxã (Revuelta, 1998). Outro ponto de discussão é o papel que as novas tecnologias poderiam representar no processo de avaliação por pares, aportando soluções por um lado e colocando novos problemas (Sabbatini, 1998). No entanto, as publicações eletrônicas, mesmo que adotem processos de análise e certificação de qualidade, tendem ainda a serem consideradas de menor prestígio do que as publicações tradicionais (Butler, 1994), dificultando o processo de sua afirmação 
dentro do modelo de publicação acadêmica.

Assim, a publicação eletrônica, por constituir um método rápido de difundir os resultados para a comunidade científica faz com que o papel desempenhado pela revisão por pares, um dos elementos fundamentais do sistema de publicações científicas, seja questionado frente a novos modelos de verificação da qualidade da informação.

Além disso, cabe ressaltar que o sistema de revisão tradicional, segundo alguns autores, já se apresenta à beira do colapso devido ao número crescente de artigos submetidos e devido à especialização crescente. Alguns dos problemas potenciais com a revisão por pares são intensificados pela lentidão dos sistema postais. Atrasos no envio e recebimento de cartas dificultam a resolução de problemas entre autores e revisores. A utilização das redes de computadores, por outro lado, desde que aliada a uma série de procedimentos claro, pode reduzir o tempo de "incubação" total entre a submissão de um manuscrito e sua publicação, reduzindo também os danos causados por práticas de revisão inadequadas.

\begin{abstract}
Não existem diferenças essenciais entre a mídia em papel e eletrônica com respeito à análise por pares. E a Net é populada por seres humanos frágeis, da mesma forma que o mundo do papel é. Mas a Net oferece a possibilidade de se distribuir as cargas da análise por pares de forma mais eqüitativa, selecionando árbitros em uma base mais ampla e sistemática (revisões eletrônicas da literatura, análises de citação, até mesmo com a submissão de chamadas para revisores em bulletins boards especialistas pertinentes e permitindo aqueles com tempo de se apresentarem como voluntários). A velocidade com que o manuscrito pode ser circulado eletronicamente também é uma vantagem, assim como a conveniência de muitos estão descobrindo em ler e comentar os manuscritos exclusivamente com a leitura em tela. Ao final de tudo, implementar o sistema tradicional de revisão por pares eletronicamente somente não é somente iminentemente possível, mas poderá ser a solução ótima, até mesmo com editores de revistas em papel preferindo conduzir a arbitragem em meio eletrônico. (Harnad, 1996)
\end{abstract}

Desta forma, a validade da revisão por pares é independente do meio em que é realizada, embora o meio eletrônico possa baratear e aumentar a eficiência do processo. Se todos artigos fossem submetidos eletronicamente, Varian (1998) estima que os custos de publicação seriam reduzidos em $50 \%$ devido à redução dos custos de trabalho administrativo, envio, realização de fotocópias, entre outros. Neste cenário, foi desenvolvido o projeto Electronic Submission and Peeer Review (ESPERE), dentro do marco do projeto elib - Electronic Libraries Programme, com o objetivo de investigar a possibilidade de se automatizar o processo de revisão, através do uso extensivo do correio eletrônico, da utilização do PDF como formato preferido para a submissão eletrônica de manuscritos e do desenvolvimento de interfaces amigáveis a serem utilizadas tanto pelos autores como pelos revisores (Electronic Submission and Peeer Review Project - ESPERE, 1997).

Porém, ao mesmo tempo em que a análise por pares é amplamente reconhecida como mecanismo essencial para distinguir entre o material científico de confiança e materiais de menor nível, novos sistemas estão sendo propostos para se tomar plena vantagem do novo meio. Uma das alternativas possíveis seria através de "avaliações post-hoc" de artigos "publicados" na Internet. Estas avaliações poderiam variar de julgamentos informais de cientistas navegando pela Internet a mecanismos formais e estruturados para organizar, avaliar e filtrar a informação disponível. Outra linha seria a adoção de revistas "interativas" envolvendo comentários e mesmo debates contínuos sobre artigos e questões emergentes.

Outros autores propõe a revisão por pares aberta, ou "open peer review" onde autores como Nadasdy (1997) e revisores não contariam com o caráter tradicional da anonimidade. Estes sistemas poderiam funcionar através do auto-policiamento de autores ou através da publicação dos artigos não revisados, deixando aos leitores a tarefa de 
avaliação. Uma variante deste último processo está sendo adotada pelo British Medical Journal que pretende utilizar os comentários adicionados livremente pelos leitores como base para a publicação. Parker (1998) alertam para o fato de que estes sistemas poderiam ser manipulados por autores de forma que estes levassem determinada vantagem no processo de avaliação, além de outras questões relacionadas com a implementação prática deste tipo de propostas. Além disso, o conceito de autoria também estaria sendo colocado em cheque (Grusin, 1994; Antonio, 1998), com os papéis seqüenciais de autor e leitor misturando-se simultaneamente para o surgimento do criador/compilador/comentador. Uma vez que estes papéis intermediários teriam menos autoridade, o sistema atual de avaliação e reconhecimento também teria que ser adaptado a esta nova realidade, tarefa que não se apresenta nem simples nem rápida.

Harnad (1996) sugere um sistema em que um autor submeteria um "artigo de trabalho" na Internet que seria comentado por qualquer pessoa interessada, seguida por um processo rigoroso de seleção que levaria em conta os comentários realizados durante a fase de revisão por pares aberta. Este processo é batizado de "scholarly skywritting", ou seja discussão interativa em tempo real, mais rápida e aberta que o processo de revisão tradicional, mas ao mesmo tempo mais reflexiva que um simpósio face-a-face.

Neste sentido, o "scholarly skywritting" consistiria a quarta revolução cognitiva na história da humanidade. Baseando-se no trabalho de Marshal Mcluhan (1962) e ele mesmo um cientista cognitivo, Harnad descreve a primeira revolução cognitiva com o surgimento da linguagem; a segunda revolução viria com o advento da escrita; a terceira revolução com a invenção da imprensa. Estas transformações midiáticas são consideradas revolucionárias na medida em que alteram a forma como nos comunicamos e tiveram um efeito qualitativo sobre como pensamos. A fala permitiu estabelecer proposições, a escrita permitiu preservá-las e a imprensa permitiu preservá-las independentemente do criador original. Harnad (1991) propõe que a quarta revolução cognitiva estaria sendo operada pelas redes de computadores, com discussão interativa em tempo real assumindo uma nova forma de comunicação caracterizada por uma nova relação de interação com a informação e de produção do conhecimento. Este novo universo comunicacional também é denominado por Harnad como a "Galáxia PósGutenberg". Apesar destas propostas inovadoras, Harnard (1997) também assume uma posição de cautela, ressaltando a diferença entre a revisão por pares e o comentário por pares, alegando que este último apesar de constituir um complemento valioso para a revisão não poderia nunca atuar como substituto. Os pareceres dos revisores, escritos somente para o editor, não se assemelham de qualquer forma aos comentários destinados se tornarem públicos a toda uma comunidade acadêmica.

A importância da distinção entre discussão e publicação não deve ser subestimada. A publicação que tem como função preservar o registro da ciência possui um caráter "sagrado" [2], ao estabelecer o conhecimento científico. Em uma sociedade cada vez mais dependente da ciência e da tecnologia, as decisões baseadas em conhecimento científico e tecnológico possuem cada vez mais importância, de forma que a qualidade da informação se torna uma questão crucial no atual. Tome-se como exemplo o caso de termos um parente diagnosticado com uma doença grave, porém curável. Seria preferível tomar decisões sobre o tratamento baseado na literatura médica revisada ou em base de uma literatura não filtrada onde a "distinção entre conhecimento fiável e ignorância, incompetência ou charlatanismo é deixada inteiramente a cargo do leitor?" (Harnad, 1998).

\section{Questões de integridade, legitimidade e privacidade}

Uma questão que tem assumido grande importância com as redes de computadores é o problema de se manter a privacidade pessoal neste meio. Assim, para que a confiança que 
os editores científicos construíram no meio acadêmico seja mantida é necessário reassegurar este papel a autores e a usuários, disponibilizando informações sobre quais dados são coletados e o que se faz com tais dados. Mais do que isso, a U.S. General Services Administration recomenda as seguintes ações em relação à privacidade e que deveriam ser adotadas pelos editores científicos na Internet: priorizar a proteção da privacidade pública em sítios WWW, manter-se a par dos impactos que a tecnologia possui sobre a privacidade, notificar o público no caso de se efetuar coleta de dados de usuário e proteger a informação coletada (Turner, 1998). O conjunto de princípios mais compreensivo lidando com informação pessoal foi publicado pela OCDE em 1981, que provê limites para a obtenção de dados pessoais, assim como de seu uso. Ainda que estes princípios tenham sido utilizados como modelo para a legislação americana a nível federal e estadual, os Estado Unidos estão longe de garantir a privacidade individual em meios eletrônicos (Goodyear, 1993). Paul e Gochenouer (1994) analisam alguma das propostas do governo norte-americano em relação à privacidade das telecomunicações e suas possíveis implicações.

Outra questão de âmbito ético e legal que surge com a publicação eletrônica é a de se a fraude científica irá aumentar com sua adoção. Por um lado o meio eletrônico poderá permitir novas e mais eficientes formas de fraude, como também poderá fazer o processo de publicação mais completo e transparente, reduzindo o problema. Assim pelo lado negativo, no meio eletrônico poderia existir uma maior facilidade em se copiar e fabricar dados, alterar dados de outros, manipular imagens ou disfarçar tentativas de engano depois que surgissem suspeitas [3]. Porém, a simples disponibilidade ampla dos computadores nas atividades de pesquisa, por si só, já facilitaria tais atividades, mesmo com a permanência da publicação em papel. A constatação de nenhum sistema poderia erradicar a fraude na ciência é mitigada, por outro lado, pela promessa de vários modos através dos quais a publicação científica poderia aumentar a integridade do arquivo científico. Por exemplo, artigos científicos poderiam ser publicados juntamente com os dados originais de pesquisa e com os softwares de análise, todas as discussões realizadas no processo de revisão por pares poderiam ser publicadas, reassegurando a integridade do processo; correções poderiam ser publicadas quase que imediatamente; a não limitação de espaço permitiria uma melhor descrição dos métodos utilizados; na medida em que mais textos completos fossem disponibilizados em bases de dados seria mais difícil publicar os mesmos dados mais de uma vez, evitando redundância e finalmente, a vinculação entre artigos através das referências bibliográficas faria mais fácil identificar casos em que a as "referências de suporte" não apoiam realmente o estudo. Devido ao caráter primitivo da publicação eletrônica, as questões sobre a integridade da informação estão em fase de formulação ainda, embora espere-se que novos métodos de preservar, e mesmo aumentar, a integridade da informação serão desenvolvidos (Smith, 1998).

Por outro lado, uma das principais dificuldades envolvidas na transição do modelo impresso para o modelo em papel seria o de manter a integridade da informação em um ambiente de rede. Neste sentido, a publicação impressa possui uma percepção de robustez maior, que é no entanto difícil de justificar objetivamente dadas as tecnologias e práticas de publicação correntes. Suplantar ou suplementar substancialmente a integridade do sistema de publicações em papel será uma questão mandatória para que um futuro sistema de publicações eletrônica seja adotado (Lynch, 1996). Kling e McKim (1998) alertam para o fato de que as diferenças disciplinares no uso e caráter da publicação eletrônica são moldadas socialmente pela práticas e estruturas deste campo em questão. Neste sentido, diferentes disciplinas constróem a confiança nas publicações de forma diferenciada, podendo depender de fatores como a centralização relativa do controle e fomento das atividades científicas do campo, do grau de integração industrial, do papel exercido pela cultura oral nesta comunidade e pelo grau de concentração de canais de 
Ao contrário dos materiais impressos que possuem um certo caráter fixo e finito, os textos publicados eletronicamente, e especialmente na Internet, possuem um caráter fluido. Assim, tanto autores quanto leitores podem facilmente alterar o tamanho e a tipografia dos documentos. Do ponto de vista do autor, exclusivamente, parágrafos podem ser rearranjados, palavras inseridas, gráficos inseridos, excluídos ou redimensionados. Da mesma forma, novos vínculos podem ser incluídos e vínculos antigos podem ficar desatualizados na medida em que os documentos aos quais se relacionam "desaparecem".

O sistema de publicação impressa possui quatro qualidades percebidas em relação à integridade (Lynch, 1996): resistência a mudanças de versão não anunciadas e não identificáveis, ou seja, assume-se que a publicação em papel cria um arquivo permanente de uma versão de um trabalho. Neste sentido, a publicação impressa depende das características da produção em massa de um grande número de objetos idênticos e a disponibilidade de uma versão revisada de um trabalho depende de um novo ato de publicação. A distribuição eletrônica, por outro lado, principalmente quando não está associada a um meio físico de distribuição como o CD-ROM, é percebida como um meio em que versões podem ser revisadas e atualizadas sem qualquer notificação. Esta poderia ser uma vantagem da publicação eletrônica pois alguns projetos caracterizados pela publicação contínua de edições revisadas poderiam ser transformados em bases de dados permanentemente atualizadas. No entanto, um trabalho desta natureza é culturalmente oposto à visão do registro científico como constituído de uma série de contribuições discretas, permanentemente fixadas e de autoria facilmente determinável. Para solucionar este problema deverão ser desenvolvidas tecnologias de controle de versões e edições, baseadas em técnicas de adição de marcas d'água eletrônicas a objetos digitais. Além disso, a adoção de políticas de administração adequadas, normas estabelecidas para autores e editores e estruturas e protocolos de citação possibilitará a retenção do histórico de publicação de uma obra.

A segunda qualidade percebida seria a da confiança nos canais de distribuição. No meio impresso, tipicamente se assume que as coisas são o que aparentam ser e raramente a validade de uma revista recebida através do correio é questionada. Esta credibilidade da matéria impressa baseia-se fundamentalmente na suposição de que esta informação seria de difícil falsificação (o que com o atual nível de tecnologia de impressão e digitalização não é mais verdade). No ambiente de rede, por sua vez, sempre se questiona se os arquivos vêm realmente e se são realmente cópias do que o editor distribuiu. A definição e implantação de padrões de verificação de origem de documentos na rede envolveria a questão de propriedade intelectual e de restrição de exportação de vários algoritmos criptográficos, ainda que estas técnicas apresentem-se bem desenvolvidas. Neste caso específico, a publicação eletrônica estaria sendo submetido a critérios mais rigorosos do que os aplicados ao modelo tradicional.

Finalmente, no meio impresso o ato da publicação implica em dar controle das cópias aos indivíduos e às bibliotecas. Assim, o princípio legal da "primeira venda" (ou seja, uma vez que um indivíduo ou organização adquira uma cópia de um trabalho impresso, ele possui autoridade sobre o objeto) suporta a integridade do sistema de publicação impressa como um meio de assegurar o registro histórico do discurso. Os leitores podem auditorar este arquivo através da consulta os materiais publicados em bibliotecas e estas providenciam anonimidade a este acesso. Uma pessoa pode inspecionar os trabalhos publicados independentemente de que o editor ou autor da obra tenha conhecimento deste ato. No ambiente de informação distribuída em rede nenhuma destas forças possui paralelos e o ato de publicação não é bem definido, assim como não o é a responsabilidade de reter e prover acesso a longo prazo às várias versões "publicadas" de 
uma obra. A título de conclusão, Lynch nota que algumas das imposições feitas em relação a integridade das publicações eletrônicas são muito superiores às que o sistema de publicações impressas pode fornecer. Porém, à medida em que migrarmos para um ambiente de informação disponível em rede e nos familiarizarmos com este novo meio, algumas destas expectativas serão abrandadas por uma dose de realismo.

\section{Percepção das publicações científicas eletrônicas}

Segundo a filosofia da tecnologia, qualquer inovação tecnológica para que seja realmente adotada deve satisfazer às necessidades de seus entornos econômicos, sociais e políticos (Quintanilla, 1989). Neste sentido, a adoção do modelo eletrônico depende em grande medida da percepção que seus possíveis usuários tenham dela; em outras palavras da percepção que a própria comunidade acadêmica tenha das revistas científicas na Internet. A utilidade e valor da informação disponibilizada pelas revistas científicas apresenta-se alta para quaisquer padrões de uso. Assim, é imperativo que a transformação da informação a partir da revista em papel para o modelo eletrônico de disseminação mantenha ou aumente os atributos da comunicação e continue a servir todos padrões de uso e busca da informação que levem a resultados benéficos por parte da comunidade científica (Tenopir \& King, 1998).

Apesar de que a facilidade de acesso seja apontada extensivamente como uma das principais vantagens da publicação eletrônica, por outro lado, esta também possui seus problemas característicos, como por exemplo, as quedas de energia, softwares com desempenho irregular, falhas de sistema, quedas na rede e existência de vírus de computador. No momento, reclamações em relação à congestão da rede e problemas de largura de banda são comuns (Resh, 1998).

Já a serendipidade nas revistas eletrônicas não seria suportada no mesmo modo que nas revistas impressas, pois a assinatura implica automaticamente em uma variedade de conteúdo maior (Silverman, 1996). Dessa forma, mais e mais, no meio eletrônico se lê somente a informação desejada, devido aos mecanismos sofisticados de localização e recuperação. $\mathrm{O}$ folheamento de material tradicional pode levar a descoberta de dados pelos quais não se estava buscando originalmente. A pesquisa teórica, particularmente, consiste no desenvolvimento de técnicas para solucionar problemas particulares e freqüientemente estas técnicas podem ser aplicadas a problemas completamente diferentes (Landshoff, 1998).

A principal questão de percepção para Resh (1998) seria a de que a leitura das revistas científicas é realizada em maior parte por pesquisadores jovens, enquanto que as decisões de como estas revistas comunicam a informação é realizada por editores mais velhos. Assim, as mudanças do meio estão sendo realizadas de acordo com o que os produtores, ao invés dos consumidores, necessitam, esperam e estão prontos a usar. A reputação e percepção das revistas eletrônicas, segundo esta visão, tenderá a aumentar pois os cientistas mais jovens tendem a ver a publicação eletrônica como uma solução para problemas como o crescimento exagerado do preço de assinaturas e no atraso das publicações, ao mesmo tempo em que permite novas dimensões do discurso científico (Abate, 1997).

Apesar disso, a percepção das revistas eletrônicas estaria assumindo um caráter mais positivo, antes do que o esperado. Um estudo realizado pela Sociedade Max Planck (MPG), na Alemanha, com a finalidade de investigar a viabilidade e usabilidade dos recursos eletrônicos para fins de pesquisa, documentou uma mudança majoritária no uso da informação de pesquisa acadêmica. Os dados obtidos demonstraram que o acesso à rede, as revistas eletrônicas e mesmo a publicação no meio eletrônico assumem um caráter de grande importância para o pesquisador de alto nível. O estudo revelou uma 
grande aceitação das revistas eletrônicas e uma relutância em se voltar ao formato impresso exclusivamente. A pesquisa também identificou vantagens e desvantagens das publicações eletrônicas. O acesso a partir da mesa de trabalho, a atualidade da informação, a facilidade de se copiar artigos e os métodos adicionais de busca e localização da informação foram identificados como as principais vantagens. Por outro lado, a ausência de arquivamento como ameaça ao acesso continuado, a inexistência de números antigos, a necessidade da leitura em tela e a dependência em relação ao acesso à rede foram identificadas como as principais desvantagens (Rusch-Feja, 1999).

Já a questão da promoção e avaliação constitui um dos tópicos mais intensamente debatidos no ambiente acadêmico. Em resposta a uma demanda social por uma maior verificabilidade de todos elementos constituintes do sistema de educação superior, as recomendações de promoção e avaliação estão sofrendo, ao redor do mundo, um reexame mais crítico. Em relação às publicações eletrônicas, uma das grandes questões seria a de se este tipo de publicação seria levado em conta nos processos de promoção e avaliação acadêmicas, possuindo ou não o mesmo status da publicação em papel. Um estudo tentando examinar os efeitos das publicações eletrônicas sobre a comunicação científica em termos de promoção e avaliação realizado por Cronin e Overfelt (1995) revelou que o meio acadêmico em um primeiro momento, ou seja, entre 1988 e 1994, mostra-se relutante em relação à credibilidade das revistas eletrônicas em comparação com seus congêneres em papel.

Langston (1996), por sua vez, identificou seis aspectos relacionados com a publicação acadêmica online que afetam os processos de avaliação acadêmica: o grau de presença de produtores, editores e consumidores na WWW; a percepção da academia em relação a estabilidade e qualidade das publicações eletrônicas; 0 fato das publicações eletrônicas possuírem ou não revisão por pares; a existência de diferentes atitudes em relação à tecnologia, conforme disciplina científica; as questões da coexistência de publicação em formato impresso e eletrônico e de se o conteúdo varia entre formas e finalmente a facilidade de acesso, incluindo-se aí o acesso à tecnologia computacional adequada e a habilidade de se recuperar informação relevante através de índices e outras ferramentas. O caráter recente do estabelecimento das publicações eletrônicas também seria outro motivo que levaria a um menor prestígio ou reconhecimento deste formato de publicação.

Cronin e Overfelt (1995) notam ainda outras preocupações de autores em relação à receptividade das publicações eletrônicas, das quais podemos destacar a falta de cobertura pelas fontes de informação secundárias, a integridade das publicações e a questão do arquivamento. A realização de uma pesquisa por correio com catedráticos de 168 departamentos acadêmicos em universidades norte-americanas teve como objetivo verificar em que extensão os comitês de avaliação "reconhecem e premiam a excelência em se publicar informação em ambiente de rede" e se os mesmos padrões e critérios são utilizados na avaliação de publicações impressas e eletrônicas. $\mathrm{O}$ estudo concluiu que a questão do meio de publicação não consiste um ponto importante no que concerne as decisões de promoção e avaliação, embora sugiram que exista o potencial de haver inconsistências de interpretação e prática. Já um estudo realizado por Neely (1999) em relação aos processos utilizados na avaliação de bibliotecários de 113 bibliotecas constituintes da Association of Research Libraries conclui que o cenário se mostra promissor para a publicação eletrônica como base para a avaliação acadêmica.

\section{Bibliografia}

ABATE, T (1997): Publishing scientific journals on-line. Bioscience, 47, 1997. p. 175179. Apud: RESH, V. H.(1998): Science and communication: an author/editor/user's perspective on the transition from paper to electronic publishing. [online]. Issues in 
Science and Technology Librarianship, summer. Available from World Wide Web: <http://www.library.ucsb.edu/istl/98-summer/article3.html>. [04/10/99].

ANTONIO, I. (1998). Autoria e cultura na pós-modernidade. [online]. Revista da Ciência da Informação. Brasília, v. 27, n. 2. Available from World Wide Web: <http://www.ibict.br/cionline/artigos/2729812.pdf>. [30/10/98].

BUTLER, H. J. (1994): Where does scholarly electronic publication get you?. [online]. Journal of Scholarly Publishing, v. 26, n. 4, p. 174-186. Apud: LANGSTON, L. (1996): Proceedings of Untangling the Web, University of California. Available from World Wide Web: <http://www.library.ucsb.edu/untangle/langston.html>. [14/10/98].

CARNEVALE, D. (1999): Web services help professors detect plagiarism. [online]. The Chronicle of Higher Education, nov. Available from World Wide Web: <http://www.chronicle.com/free/v46/i12/12a04901.htm>. [05/12/99].

CRONIN, B., OVERFELT, K.(1995): E-Journals and Tenure. Journal of the American Society for Information Science, 46, p. 700-703. Apud: NEELY, T. I. (1999): The impact of electronic publications on promotion and tenure decisions. [online]. Leading Ideas: Issues and Trends in Diversity, Leadership and Career Development, n. 10. Available from World Wide Web: <http://www.arl.org/diversity/leading/issue10/tneely.html>. [15/10/99].

ELECTRONIC SUBMISSION AND PEEER REVIEW PROJECT - ESPERE (1997). Annual Report. April 1996-June 1997. [online]. Relatório técnico apresentado ao elib Electronic Libraries Programme. Available from World Wide Web: $<$ http://www.espere.org/annrpt.pdf>. [05/12/99].

GOODYEAR, M. L.(1993): Information policy for electronic information resources. [online]. The Public-Access Computer Systems Review, v.4, n. 6, p. 23-31. Available from World Wide Web: <http://info.lib.uh.edu/pr/v4/n6/goodyear.v4n6>. [30/09/98].

GRUSIN, R.(1994): What is an electronic author? Theory and the technological fallacy. [online]. Configurations, v. 2, n. 3, p. 469-483. Apud: LANGSTON, L. (1996): Proceedings of Untangling the Web, University of California. Available from World Wide Web: <http://www.library.ucsb.edu/untangle/langston.html>. [14/10/98].

HARGENS, L. L.(1990): Variations in journal peer review systems: possibles causes and consenquences. Journal of the American Medical Association, n. 263, p. 13481352.Apud: HARNAD, S. (1996): Implementing peer review on the Net: scientific quality control in scholarly journals. En: PEEK, R.P., NEWBY, G.B. (eds.). Scholarly publishing: the electronic frontier. Cambridge: MIT Press, 1996. 363 p. p.103-118.

HARNAD, S. (1990): Scholarly skywriting and the prebublication continuum of scientific inquiry. [online]. Psychological Science, 1, p. 342-344. Available from World Wide Web: $<\mathrm{ftp}$ ://ftp.princeton.edu/pub/harnad/Harnad/HTML/harnad90.skywriting.html>. [14/10/98].

HARNAD, S. (1991):. Scholarly skywritting and the prepublication continuum of scientific inquiry. [online]. Psychological Science,1, p. 342-343. Available from World Wide Web:<ftp://princeton.edu/pub/Harnad/harnad90.skywritting>. [13/10/98]. (b).

HARNAD, S. (1997). Learned inquiry and the Net: the role of peer review, peer commentary and copyright. [online]. Antiquity, 71. p. 1042-1048. Available from World Wide Web: 
<ftp://ftp.princeton.edu/pub/harnad/Harnad/HTML/harnad97.antiquity.html>. [14/10/98]. (a).

HARNAD, S. (1998): The invisible hand of peer review. [online]. Nature, 5 de novembro de 1998, Web Matters Section. Available from World Wide Web: <http://helix.Nature.com/webmatters/invisible/invisible.html>. [14/10/98]. (a).

KLING, R., LAMB, R.(1996): Analyzing alternate visions of electronic publishing and digital libraries. In: PEEK, R.P., NEWBY, G.B. (eds.). Scholarly publishing: the electronic frontier. Cambridge: MIT Press. 363 p. p. 17-54.

LANDSHOFF, P.(1998): The traditional journal. [online]. In: BUTTERWORTH, I. (ed.). The impact of electronic publishing on the academic community: an international workshop organized by the Academia Europaea and the Wenner-Gren Foundation. Londres: Portaland Press. Available from World Wide Web: <http://tiepac.portlandpress.co.uk/books/online/tiepac/session3/ch1.htm>. [17/10/99].

LANGSTON, L. (1996): Scholarly Communication and electronic publication: implications for research, advancement and promotion. [online]. Proceedings of Untangling the Web, University of California. Available from World Wide Web: <http://www.library.ucsb.edu/untangle/langston.html>. [14/10/98].

McLUHAN, H. M. (1962): The Gutenberg Galaxy: The Making of Typographic Man, University of Toronto Press.

MERTON, P. (1968): Las pautas de conductas de los científicos. In: La sociología de la ciencia, v. 2. Madrid: Alianza, 1973.

LEDERBERG, J. (1996): Options for the future. [online] D-Lib Magazine, maio. Available from World Wide Web: <http://www.dlib.org/dlib/may96/05lederberg.html>. [14/10/98].

LYNCH, C. A (1996). Integrity issues in electronic publishing. In: PEEK, R.P., NEWBY, G.B. (eds.). Scholarly publishing: the electronic frontier. Cambridge: MIT Press. 363 p. p.133-146.

NADASDY, Z. (1997): Electronic Journal of Cognitive and Brain Sciences: a truly allelectronic journal: let democracy replace peer review. [online]. The Journal of Electronic Publishing, v. 3, n. 1. Available from World Wide Web: <http://www.press.umich.edu:80/jep/03-01/EJCBS.html>. [05/10/99].

NEELY, T. I. (1999) The impact of electronic publications on promotion and tenure decisions. [online]. Leading Ideas: Issues and Trends in Diversity, Leadership and Career Development, n. 10. Available from World Wide Web: <http://www.arl.org/diversity/leading/issue10/tneely.html>. [15/10/99].

PARKER, R. (1998): Quality control in electronic publication. [online]. Trabalho apresentado no AAAS/UNESCO/ICSU Workshop on Developing Practices and Standards for Electronic Publishing in Science, UNESCO, Paris, 12-14 de outubro de 1998. Available from World Wide Web: http://www.aaas.org/spp/dspp/sfrl/projects/epub/ses3/parker1.htm> [11/09/99].

PAUL, M. J, GOCHENOUER, J. E. (1994): Telecommunications, isolation, and the erosion of privacy. [online]. Interpersonal Computing and Technology: An Electronic 
Journal for the 21st Century, v. 2, n. 3, p. 82-98. Available from World Wide Web: <http://www.helsinki.fi/science/optek/1994/n3/paul.txt>. [22/11/98].

PRIMO-YÚFERA, E (1994): Introducción a la investigación científica y tecnológica. Madrid: Alianza Editorial.

QUINTANILLA, M.A. (1989): Problemas filosóficos de la tecnología. In: Tecnología un ensayo filosófico. Madrid: FUNDESCO. p. 19-38.

RESH, V. H. (1998): Science and communication: an author/editor/user's perspective on the transition from paper to electronic publishing. [online]. Issues in Science and Technology Librarianship, summer edition. Available from World Wide Web: <http://www.library.ucsb.edu/istl/98-summer/article3.html>. [04/10/99].

REVUELTA, G. (1998): La revisión revisable. Congreso Internacional sobre publicaciones biomédicas basadas en el sistema peer review y comunicación global. [online]. Barcelona, Quark - Ciencia, Medicina, Comunicación y Cultura, n. 10. Available from World Wide Web: <http://www.imim.es/quark/num10/cronica.htm>. [03/06/99].

RUSCH-FEJA, D. (1999) Evaluation of usage and acceptance of electronic journals. Results of an electronic survey of Max Planck Society researchers including usage statistics from Elsevier, Springer and Academic Press. [online]. D-Lib Magazine, n. 10, v. 5. Available from World Wide Web: <http://www.dlib.org/dlib/october99/ruschfeja/10rusch-feja-summary.html>. [17/10/99].

SABBATINI, M. (1998): A Internet Como Ferramenta de Qualidade Científica. [online]. Mídia Fórum, São Bernardo do Campo, v. 3. n. 8. Available from World Wide Web: <http://www.webpraxis.com/msabba/mf001.htm>. [10/09/98].

SILVERMAN, R. J. (1996): The impact of electronic publishing on the academic community. In: PEEK, R.P., NEWBY, G.B.(eds.). Scholarly publishing: the electronic frontier. Cambridge: MIT Press. 363 p. p. 55-69.

SMITH, R. (1998): Maintaining the integrity of electronic publications: potential problems and possible solutions I. [online]. Trabalho apresentado no AAAS/UNESCO/ICSU Workshop on Developing Practices and Standards for Electronic Publishing in Science, UNESCO, Paris, 12-14 de outubro de 1998. Available from World Wide Web: <http://www.aaas.org/spp/dspp/sfrl/projects/epub/ses3/smith.htm> [11/09/99].

STANEK, W. R., PURCELL, L. (1995): Electronic Publishing Unleashed. Sams Publishing.

TENOPIR, C., KING, D. W. (1998): Designing electronic journals with 30 years of lessons from print. [online]. The Journal of Electronic Publishing, v. 4, n. 2. Available from World Wide Web:

$<$ http://www.press.umich.edu/jep/04-02/king.html>. [03/12/98].

TURNER, J. A. (1998): Privacy in e-pub: smoke and mirrors. [online]. Trabalho apresentado no AAAS/UNESCO/ICSU Workshop on Developing Practices and Standards for Electronic Publishing in Science, UNESCO, Paris, 12-14 de outubro de 1998. Available from World Wide Web: <http://www.aaas.org/spp/dspp/sfrl/projects/epub/ses5/turner.htm> [11/09/99]. 
VARIAN, H. (1998): The future of electronic journals. [online]. The Journal of Electronic Publishing, v. 4, n. 1. Publicado originalmente sob o título Future of electronic journals: some especulations about the evolution of academic electronic publishing, apresentado na Scholarly Communication and Technology Conference, Emory University, Atlanta, abr. 1997. Available from World Wide Web: <http://www.press.umich.edu/jep/0401/varian.html>. [13/10/99].

\section{NOTAS}

[1] Por publicação eletrônica, entende-se qualquer tecnologia de distribuição de informação em uma forma que possa ser acessada e visualizada pelo computador e que utiliza recursos digitais para adquirir, armazenar e transmitir informação de um computador para outro (Stanek \& Purcell, 1995).

[2] Lederberg (1996) argumenta que uma publicação acadêmica consiste em uma "afirmação sob juramento". Mentir em uma publicação consistiria em uma forma de "perjúrio", passível de sanções legais.

[3] Carnevale (1999) relata a experiência de algumas tecnologias utilizadas na detecção de plágio de trabalhos escolares.

(C) Ediciones Universidad de Salamanca. 